\title{
Protein profiling and functional analysis of liver mitochondria from rats with nonalcoholic steatohepatitis
}

\author{
YANTING YOU ${ }^{1,2^{*}}$, YUXING ZHANG ${ }^{1,2^{*}}$, YUANYUAN LU ${ }^{1,2}$, KEKE HU $^{1,2}$, \\ XIAOHU QU ${ }^{1,2}$, YONGZHAG LIU ${ }^{1,2}$, BIN LU $^{1,2}$ and LIQIN JIN ${ }^{1-3}$ \\ ${ }^{1}$ Key Laboratory of Laboratory Medicine, Ministry of Education, and Zhejiang Provincial Key Laboratory \\ of Medical Genetics; ${ }^{2}$ School of Laboratory Medicine and Life Science; ${ }^{3}$ School of Basic Medical Sciences, \\ Wenzhou Medical University, Wenzhou, Zhejiang 325035, P.R. China
}

Received April 7, 2016; Accepted March 6, 2017

DOI: $10.3892 / \mathrm{mmr} .2017 .6893$

\begin{abstract}
Mitochondrial dysfunction is closely associated with the pathogenesis of nonalcoholic steatohepatitis (NASH). The aim of the present study was to comprehensively determine mitochondrial abnormalities in NASH by detecting the proteomics in liver mitochondria in a NASH rat model, which was induced for 16 weeks by the provision of a high fat and high cholesterol diet (HFD). Serum parameters, including triglycerides, total cholesterol, low-density lipoprotein cholesterol and high-density lipoprotein cholesterol were determined, and hematoxylin and eosin staining of liver tissues was examined
\end{abstract}

Correspondence to: Professor Liqin Jin, School of Basic Medical Sciences, Wenzhou Medical University, 7 a209 Chashan Campus, Chashan University Town, Wenzhou, Zhejiang 325035, P.R. China E-mail: liqinjinwmu@139.com

Professor Bin Lu, School of Laboratory Medicine and Life Science, Wenzhou Medical University, 4b 521 Chashan Campus, Chashan University Town, Wenzhou, Zhejiang 325035, P.R. China

E-mail: lubmito@wmu.edu.cn

*Contributed equally

Abbreviations: NASH, nonalcoholic steatohepatitis; HFD, high fat and high cholesterol diet; iTRAQ, isobaric tags for relative and absolute quantitation; 2D LC-MS/MS, two-dimensional liquid chromatography-tandem mass spectrometry; KEGG, Kyoto Encyclopedia of Genes and Genomes; NAFLD, nonalcoholic fatty liver disease; HCC, hepatocellar carcinoma; ROS, reactive oxygen species; ATP, adenosine triphosphate; SCD, standard chow diet; TC, total cholesterol; TG, triglyceride; LDL-C, low-density lipoprotein; HDL-C, high-density lipoprotein; H\&E, hematoxylin and eosin; RT-qPCR, reverse transcription-quantitative polymerase chain reaction; NADH, ubiquinone oxidoreductase; CS, citrate synthase; MMP, mitochondrial membrane potential; mtDNA, mitochondrial DNA; MRC, mitochondrial respiratory complex; ACSM2, acyl CoA synthetase; FPP, farnesyl pyrophosphate

Key words: mitochondrial function, nonalcoholic steatohepatitis, isobaric tags for relative and absolute quantitation, rat model to evaluate the NASH rat model. Various parameters associated with mitochondrial function were examined, including mitochondrial DNA (mtDNA) copy number, mitochondrial membrane potential (MMP) and mitochondrial respiratory chain complex (MRC) activity. The mitochondrial proteomics were analyzed and identified using isobaric tags for relative and absolute quantitation labeling coupled with two-dimensional liquid chromatography-tandem mass spectrometry. The identified proteins were classified and grouped using the Blast $2 \mathrm{GO}$ program against the non-redundant protein database, the Kyoto Encyclopedia of Genes and Genomes database and the Cluster of Orthologous Groups of proteins database. Compared with the control, mtDNA copy number, MMP, and activities of MRC I and III were decreased markedly in the HFD group. A total of 18 upregulated and 13 downregulated proteins were identified, with a significant 1.2-fold difference between the control and NASH groups. The dysregulated proteins were closely involved in mitochondrial oxidative phosphorylation, the lipid metabolic process and fatty acid $\beta$-oxidation. The results of the present study provide important proteomic information regarding liver mitochondria in NASH and serve as a basis for further detailed investigations of the pathogenesis of NASH.

\section{Introduction}

Nonalcoholic fatty liver disease (NAFLD) is a common cause of chronic liver disease, and comprises a wide range of pathological changes in the liver, from non-progressive steatosis to nonalcoholic steatohepatitis (NASH), advanced fibrosis and cirrhosis, ultimately leading to hepatocellar carcinoma (HCC) (1). NASH is characterized by lobular inflammation, hepatocellular ballooning and fibrosis, and is key in the progression to cirrhosis and HCC (2). Day and James described a two-hit theory (3), regarding the hepatic triglyceride accumulation as the first hit and mitochondrial dysfunction, oxidative stress and inflammatory factors as the second hit (4). Substantial evidence has indicated that mitochondrial dysfunction is directly associated with the pathogenesis of NASH and has suggested that NASH is a mitochondrial disease $(5,6)$. Mitochondrial dysfunction impairs lipid metabolism and induces the overproduction of reactive oxygen species (ROS), 
which triggers the peroxidation of lipids and apoptosis of hepatocytes (7). In addition, this can decrease the activity of the mitochondrial respiratory chain (MRC), which further impairs adenosine triphosphate (ATP) synthesis and enhances oxidative stress (7). However, the alterations in mitochondrial proteomics in response to NASH remain to be fully elucidated.

In our previous study, a high fat and high cholesterol diet (HFD) was used to establish a NASH rat model, which is more similar to dietary conditions, compared with the methionine-choline-deficient diet in humans (8). To date, using the NASH rat model, there has been no attempt to establish a comparative proteome profile of liver mitochondria using isobaric tags for relative and absolute quantitation (iTRAQ) technology coupled with two-dimensional liquid chromatography-tandem mass spectrometry (2-D LC-MS/MS) analysis.

Compared with traditional 2-D gel electrophoresis, it is easier to analyze eight samples simultaneously using iTRAQ technology, thus enhancing throughput, and increasing sensibility and accuracy (9). To the best of our knowledge, the present study using the NASH rat model is the first to perform proteomic analysis of liver mitochondria using iTRAQ technology, to provide novel insights into the pathogenesis and progression of NASH via mitochondrial protein profiling.

\section{Materials and methods}

Animal models of NASH. Eight-week-old male Sprague-Dawley rats weighing $\sim 320 \mathrm{~g}$ were purchased from the Laboratory Animal Centre of Wenzhou Medical University (Wenzhou, China). Under a 12-h light-dark cycle and controlled temperature $\left(23 \pm 2^{\circ} \mathrm{C}\right)$, all rats were raised under specific pathogen-free conditions with free access to water and food. The animals were acclimatized to the laboratory conditions for 1 week prior to the experiments and were randomly divided into two groups: Control group $(n=6)$ and HFD group $(n=10)$. The rats in the control group and the HFD group were respectively fed with a standard chow diet (SCD) and a high fat diet (1\% cholesterol, $19 \%$ lard and $80 \%$ SCD) for 16 weeks. The body weights of the rats were measured weekly. The rats were sacrificed following overnight fasting. All protocols and procedures conformed to the guidelines of the Laboratory Animal Ethics Committee of Wenzhou Medical University, and all efforts were made in order to minimize the suffering and the number of animals used.

Blood and liver sample preparation. Following sacrifice, blood samples from the abdominal vein of the rats were collected in coagulation-promoting tubes and centrifuged at $1,500 \mathrm{~g}$ for $15 \mathrm{~min}$ at $4^{\circ} \mathrm{C}$ (Eppendorf 5810R; Eppendorf, Hamburg, Germany) to obtain the serum for biochemical analysis, which was stored at $-80^{\circ} \mathrm{C}$ prior to analysis. The levels of glucose, total cholesterol (TC), triglyceride (TG), low-density lipoprotein (LDL-C) and high-density lipoprotein (HDL-C) were measured using an automated biochemistry analyzer (Hitachi, Tokyo, Japan). The livers were excised, cleaned with ice-cold phosphate-buffered saline and weighed immediately. The left lobes of each liver were then fixed in $4 \%$ paraformaldehyde solution for further morphological analysis using hematoxylin and eosin (H\&E) staining, with images captured using a Nikon microscope (Nikon E-100 A12.0705; Nikon
Corporation, Tokyo, Japan). Additionally, sections of the liver tissue were added to a volume of storage medium containing $20 \%$ dimethyl sulfoxide, $0.21 \mathrm{M}$ mannitol and $0.07 \mathrm{M}$ sucrose ( $\mathrm{pH}$ 7.5) for mitochondrial separation, and other sections were directly snap frozen in liquid nitrogen and then preserved at $-80^{\circ} \mathrm{C}$ until use (10).

Determination of relative mtDNA copy number. Total DNA from the liver tissues was extracted using a Blood and Cell Culture DNA Mini kit (Qiagen GmbH, Hilden, Germany). Reverse transcription-quantitative polymerase chain reaction (RT-qPCR) analysis was used to determine the relative mtDNA copy number. The RT-qPCR amplification reaction was performed via SYBR-Green chemistry using Bio-Rad CFX Manage 2.1 (Bio-Rad Laboratories, Inc., Hercules, CA, USA). The mtDNA was synthesized and amplified using the following primers: $\mathrm{ND}_{1}$ forward, 5'-ATTCTAGCCACATCA AGTCTTT-3' and reverse, 5'-GGAGGACGGATAAGAGGA TAAT-3'; $\beta$-actin forward, 5'-GAAATCGTGCGTGACATT AAAG-3' and reverse, 5'-ATCGGAACCGCTCATTG-3'. Each sample was analyzed in triplicate with a $20 \mu$ l final volume containing SYBR-Green Supermix PCR 1X Master mix (Bio-Rad Laboratories, Inc.), $0.5 \mu \mathrm{M}$ forward and reverse primes and $100 \mathrm{ng}$ DNA template. Following 3 min denaturation at $95^{\circ} \mathrm{C}$, amplification was performed for 39 cycles, including $95^{\circ} \mathrm{C}$ for $10 \mathrm{sec}$ for denaturation, and $55^{\circ} \mathrm{C}$ for $30 \mathrm{sec}$ for annealing and extension. Melting curve analysis was performed at the end of each run to validate the specificity of the PCR products. The quantification of the relative mtDNA copy number was performed using the $2^{\Delta \Delta \mathrm{Cq}}$ method (11), normalized to $\beta$-actin.

Isolation of liver mitochondria. The frozen liver tissues of the rats were rapidly thawed using a preheated medium of $0.4 \%$ BSA (Beyotime Institute of Biotechnology, Haimen, China), $0.25 \mathrm{M}$ sucrose and $0.01 \mathrm{M}$ Tris- $\mathrm{HCl}\left(\mathrm{pH} \mathrm{7.5)}\right.$ at $45^{\circ} \mathrm{C}$ in a $4: 1$ ratio of medium to tissue. The mitochondria were isolated as described previously (12). In brief, the liver tissues were weighed, washed and homogenized at $4{ }^{\circ} \mathrm{C}$ in isolation buffer containing $0.5 \%$ BSA, $225 \mathrm{mM}$ mannitol, $75 \mathrm{mM}$ sucrose, $30 \mathrm{mM}$ Tris/ $\mathrm{HCl}$ and 0.5 mM EGTA (pH 7.4). The homogenate was centrifuged twice at $4^{\circ} \mathrm{C}$ and $740 \mathrm{~g}$ for $10 \mathrm{~min}$ to remove unbroken cells and nuclei, and at $4^{\circ} \mathrm{C}$ and $9,000 \mathrm{~g}$ for $10 \mathrm{~min}$ three times to precipitate the mitochondrial pellet and obtain the crude mitochondria. The pellet was resuspended in MRB buffer containing $250 \mathrm{mM}$ mannitol, $5 \mathrm{mM}$ HEPES and 0.5 mM EGTA (pH 7.4), and gently layered on top of $30 \%(\mathrm{v} / \mathrm{v})$ percoll. Following $50 \mathrm{~min}$ of centrifugation at $4^{\circ} \mathrm{C}$ and $95,000 \mathrm{x}$ g, collection and washing of the mitochondrial fraction twice, centrifugation was performed at $4^{\circ} \mathrm{C}$ and $6,300 \times \mathrm{g}$ for $10 \mathrm{~min}$ with $\mathrm{MRB}$ to remove the residual percoll. Using the BCA protein assay kit (Thermo; Fisher Scientific, Inc., Waltham, MA, USA) the concentration of mitochondrial protein was measured. Mitochondria were stored in $-80^{\circ} \mathrm{C}$ for proteomic analysis.

MRC enzymatic assays. As described above, the pellet (crude mitochondria) was resuspended in MRB and freeze-thawed three times using liquid nitrogen. All samples were measured in triplicate using a Varioskan Flash reader (Thermo Fisher Scientific, Inc.). 
The specific activity of complex I, ubiquinone oxidoreductase (NADH) was analyzed according to the decrease in absorbance at $340 \mathrm{~nm}$ due to the oxidation of NADH. The isolated mitochondria $(25 \mu \mathrm{g})$ were added into $200 \mu \mathrm{l}$ of the reaction buffer $(195 \mu \mathrm{M}$ NADH, $50 \mathrm{mM}$ potassium phosphate buffer, $10 \mu \mathrm{g} / \mathrm{ml}$ antimycin, $10 \mu \mathrm{M}$ decylubiquinone and $5 \mathrm{mM}$ $\mathrm{NaN}_{3}$ ) in a 96-well plate. Under the conditions of $340 \mathrm{~nm}$ and $30^{\circ} \mathrm{C}$, the rate of oxidation of NADH was measured for $90 \mathrm{sec}$.

The specific activity of complex II (succinate dehydrogenase) was assessed by the decrease of absorbance at $600 \mathrm{~nm}$ due to reduction of dichlorophenol indophenols. The $200 \mu \mathrm{l}$ reaction buffer $(270 \mathrm{mM}$ potassium phosphate buffer, $200 \mathrm{mM}$ succinate, $12 \mu \mathrm{M}$ rotenone, $7.5 \mu \mathrm{M} \mathrm{NaN}_{3}, 5 \mu \mathrm{g} / \mathrm{ml}$ antimycin, $100 \mathrm{mM}$ 2,6-dichlorophenolindophenolsodium salt and $20 \mu \mathrm{g}$ mitochondria protein) was equilibrated for $10 \mathrm{~min}$ at $30^{\circ} \mathrm{C}$. Following the addition of $4 \mu \mathrm{l}$ of $1 \mathrm{mM}$ decylubiquinone, the reaction was initiated and absorbance was measured at $600 \mathrm{~nm}$ for $90 \mathrm{sec}$ at $30^{\circ} \mathrm{C}$.

Complex III (decylubiquinol cytochrome coxidoreductase) was measured by the increase of absorbance at $550 \mathrm{~nm}$ due to the reduction of cytochrome $c$. The $200 \mu \mathrm{l}$ reaction buffer $(250 \mathrm{mM}$ sucrose, $100 \mathrm{mM}$ Tris/Hcl, $1 \mathrm{mM}$ EDTA, $50 \mu \mathrm{M}$ cytochrome $c, 50 \mu \mathrm{M}$ decylubiquinol, $45 \mu \mathrm{M}$ n-dodecyl- $\beta$-d-maltoside and $7.5 \mu \mathrm{M} \mathrm{NaN}_{3}$ ) was incubated for $60 \mathrm{sec}$ and the reaction was induced by adding $20 \mu \mathrm{g}$ of mitochondrial protein. The absorbance was measured at $550 \mathrm{~nm}$ for $90 \mathrm{sec}$ at $30^{\circ} \mathrm{C}$.

Complex IV (cytochrome $c$ oxidase) was measured by the decrease of absorbance at $550 \mathrm{~nm}$ due to oxidization of the reduced cytochrome $c$. The mitochondria $(20 \mu \mathrm{g})$ were added into $200 \mu \mathrm{l}$ reaction buffer, containing $9.4 \mathrm{mM}$ potassium phosphate buffer, $50 \mu \mathrm{M}$ reduced cytochrome $c$ and $450 \mu \mathrm{m}$ $\mathrm{n}$-dodecyl- $\beta$-d-maltoside. The reaction was detected at $550 \mathrm{~nm}$ for $135 \mathrm{sec}$ at $30^{\circ} \mathrm{C}$.

The activity of citrate synthase was assessed by alterations of thionitrobenzoate anion formation. The mitochondrial protein $(20 \mu \mathrm{g})$ was added to $200 \mu \mathrm{l}$ reaction buffer $(0.1 \mathrm{M}$ Tris/Hcl, 0.1 M 5,5'-dithiobis-2-nitrobenzoate, $0.3 \mathrm{mM}$ acetyl-CoA, $450 \mu \mathrm{M}$ n-dodecyl- $\beta$-d-maltoside and $500 \mu \mathrm{M}$ oxaloacetate). The absorbance was then measured at $412 \mathrm{~nm}$ for $270 \mathrm{sec}$. The activity of CS was expressed in $\mathrm{nmol} / \mathrm{min} / \mathrm{mg}$, and normalized to total tissue protein content.

ATP synthase activity. According to the manufacturer's protocol of the ATP Synthase Enzyme Activity Microplate Assay kit (Abcam, Cambridge, UK), ADP and phosphate are produced by ATP synthase hydrolyzing ATP. The oxidation of NADH is coupled with the production of ADP and ultimately becomes $\mathrm{NAD}^{+}$. The reaction was detected at $340 \mathrm{~nm}$ for $90 \mathrm{sec}$ at $30^{\circ} \mathrm{C}$.

Mitochondrial membrane potential (MMP) analysis using $J C-1$. MMP was determined in the crude mitochondria freshly isolated from liver tissues using a JC-1 Mitochondrial Membrane Potential Detection kit (Beyotime Institute of Biotechnology). According to the manufacturer's protocol, $50 \mu \mathrm{g}$ of mitochondria were stained by JC-1 and scanned at $490 \mathrm{~nm}$ excitation $/ 530 \mathrm{~nm}$ emission and at $525 \mathrm{~nm}$ excitation/590 nm emission to detect green and red JC-1 fluorescence, respectively, using the Varioskan Flash reader.
Quantitative proteomics using the iTRAQ technique. Mitochondria were solubilized in lysis buffer $(7 \mathrm{M}$ urea, $2 \mathrm{M}$ thiourea, $40 \mathrm{mM}$ Tris, $2 \mathrm{mM}$ EDTA, $1 \mathrm{mM}$ PMSF, $0.2 \% \mathrm{SDS}$ and $4 \% \mathrm{CHAPS}$ ), and sonicated at $200 \mathrm{~W}$ for $15 \mathrm{~min}$ on ice, followed by centrifugation at $4^{\circ} \mathrm{C}$ and $25,000 \mathrm{~g}$ for $20 \mathrm{~min}$. The supernatant was added to $10 \mathrm{mM}$ DTT (final concentration) and maintained at $56^{\circ} \mathrm{C}$ for $1 \mathrm{~h}$, this step was for reducing the disulfide bonds in the proteins. The mixture was kept in the dark, and $55 \mathrm{mM}$ IAM (final concentration) was added and incubated for $1 \mathrm{~h}$ in order to block the cysteines. To remove detergents, which may interfere with ITRAQ $^{\mathrm{TM}}$ labeling, the protein was precipitated by the addition of five volumes of chilled acetone for $2 \mathrm{~h}$ at $-20^{\circ} \mathrm{C}$. Following centrifugation at $4^{\circ} \mathrm{C}$ at $25,000 \mathrm{~g}$ for $20 \mathrm{~min}$, the pellet was dissolved in $500 \mu \mathrm{l}$ of $0.5 \mathrm{M}$ triethylammonium bicarbonate (Applied Biosystems; Thermo Fisher Scientific, Inc.) and sonicated again. The samples were then centrifuged at $25,000 \mathrm{~g}$ for $20 \mathrm{~min}$ at $4^{\circ} \mathrm{C}$. The Bradford method (Thermo Fisher Scientific, Inc.) was used to quantify the supernatant. Protein of each sample $(100 \mu \mathrm{g})$ was digested with trypsin (Promega Corporation, Madison, WI, USA), at 20:1 protein to trypsin ratio, overnight at $37^{\circ} \mathrm{C}$. Vacuum centrifugation was performed to dry the peptides following the digestion with trypsin. According to the $\mathrm{iTRAQ}^{\mathrm{TM}}$ reagents protocol, using 8-plex iTRAQ reagent (Applied Biosystems; Thermo Fisher Scientific, Inc.), the peptides were dissolved and samples labeled as follows: Control (CON)-2 (117 tag), CON-4 (114 tag), CON-5 (119 tag), CON-8 (116 tag), HFD-1 (113 tag), HFD-2 (118 tag), HFD-4 (115 tag) and HFD-8 (121 tag) randomly selected from the control group and HF group, respectively. Vacuum centrifugation at $4^{\circ} \mathrm{C}$ and $12,000 \mathrm{~g}$ for $10 \mathrm{~min}$ was performed to pool and dry the labeled peptide mixtures.

The peptide mixtures were added to $4 \mathrm{ml}$ solvent $\mathrm{A}$, which contained $25 \mathrm{mM} \mathrm{NaH} \mathrm{PO}_{4}$ dissolved in $25 \%$ can $(\mathrm{pH} 2.7)$ and then injected into a $4.6 \times 250 \mathrm{~mm}$ Ultremex SCX column, which worked with the LC-20AB HPLC Pump system (Shimadzu Corporation, Kyoto, Japan) and contained $5 \mu \mathrm{m}$ particles (Phenomenex, Inc., Torrence, CA, USA). A gradient of solvents was used to elute the peptides at a $1 \mathrm{ml} / \mathrm{min}$ flow rate: 10 min of $100 \%$ solvent $\mathrm{A}, 7 \mathrm{~min}$ of $5 \%$ solvent $\mathrm{B}$, which contained $1 \mathrm{M} \mathrm{KCl}$ and $25 \mathrm{mM} \mathrm{NaH}_{2} \mathrm{PO}_{4}$ dissolved in $25 \%$ $\mathrm{ACN}$ ( $\mathrm{pH} 2.7$ ), $20 \mathrm{~min}$ of $5-60 \%$ solvent $\mathrm{B}$ and $1 \mathrm{~min}$ of $60-100 \%$ buffer B. Finally, washing was performed for $1 \mathrm{~min}$ of $100 \%$ buffer B and equilibrated for $10 \mathrm{~min}$ in buffer A prior to the next loading. The absorbance of elution was monitored at $214 \mathrm{~nm}$ and the fractions were collected every $1 \mathrm{~min}$. In total, 20 fractions were collected, which were desalted in a Strata $\mathrm{X} \mathrm{C}_{18}$ column (Phenomenex, Inc.) and then dried them completely in a vacuum centrifuge.

Fractions were reconstituted with solvent A (2\% CAN, $0.1 \% \mathrm{FA}$ ) and centrifuged for $10 \mathrm{~min}$ at $4^{\circ} \mathrm{C}$ and $20,000 \mathrm{~g}$ to remove the insoluble substance, and the final concentration of peptide was adjusted to $0.5 \mu \mathrm{g} / \mu \mathrm{l}$. In each fraction, using the autosampler, $5 \mu \mathrm{g}$ of peptide mixture was injected into a Shimadzu LC-20AD nano HPLC (Shimadzu Corporation), which had a $2 \mathrm{~cm} \mathrm{C}_{18}$ trap column. Subsequently, an in-house packed analytical column $\left(75 \mu \mathrm{m} \times 10 \mathrm{~cm}, \mathrm{C}_{18}\right)$ was used to elute the peptides. The peptides were loaded at $8 \mu \mathrm{l} / \mathrm{min}$ for $4 \mathrm{~min}$ and separated at a flow of $300 \mathrm{nl} / \mathrm{min}$ over $44 \mathrm{~min}$ with a gradient of solvent B (98\% ACN and 0.1 FA). Subsequently, 
the linear gradient was increased to $80 \%$ within 2 min and maintained at $80 \%$ for $4 \mathrm{~min}$, followed by a return to $5 \%$ for $1 \mathrm{~min}$. The effluent was analyzed using a Q-Exactive mass spectrometer (Thermo Fisher Scientific, Inc.) with nanoelectrospray and voltage set at $1.6 \mathrm{KV}$. At a resolution of 7,000 in an Orbitrap mass analyzer, full MS scans were acquired from $350-2,000 \mathrm{~m} / \mathrm{z}$ for the detection. A fragment ion spectrum was produced via high-energy collision dissociation and the mass range of $100-1,800 \mathrm{~m} / \mathrm{z}$ was detected in by Orbitrap mass analyzer at a resolution of 17,500. In the MS survey scan, following a dynamic exclusion duration of $15 \mathrm{sec}, \mathrm{MS} / \mathrm{MS}$ data were obtained through data-dependent acquisition, which used the 15 most abundant precursor ions above the threshold ion count of 20,000. The automatic gain control target for full MS was $3 \mathrm{e}^{6}$ and for MS/MS was $1 \mathrm{e}^{5}$, and were used to optimize the spectra generated by the Orbitrap analyzer.

Protein identifications were performed using Discoverer 1.2 (Thermo Electron, San Jose, CA, USA), compared with a database containing the UniprotRat sequences, using the Mascot search engine (version 2.3.02; Matric Science, London, UK). In the identification of proteins, which allowed for one missed cleavage in the trypsin digestion, the tolerance of the peptide mass in MS was $20 \mathrm{ppm}$ and for fragmented ions was $0.05 \mathrm{Da}$. The conversion of $\mathrm{N}$-terminal glutamine to pyroglutamic acid, oxidation of methionine and tyrosine labeled by iTRAQ-8-plex were set as the potential variable modifications. The carbamidomethylation of cysteine at the $\mathrm{N}$-terminal of peptides and lysine labeled by iTRAQ-8-plex were considered to fix these modifications. Peptides with significance scores $(\geq 20 ; \mathrm{P}<0.01)$ were counted as identified in order to reduce false peptide identification. At least one unique peptide was involved in the identification of each confident protein and at least two unique peptides were required to quantify protein. Significant changes in the quantitative protein ratios were identified by setting cut off values of a fold change $>1.2$ and $\mathrm{P}<0.05$. Using the Blast2GO program (www.blast2go.com), compared with the non-redundant protein database (NR; NCBI), functional annotations, which had differential proteins, were performed. The Kyoto Encyclopedia of Genes and Genomes (KEGG; http://www.genome.jp/kegg/) and Cluster of Orthologous groups (http://www.ncbi.nlm.nih.gov/COG/) databases were used to classify and group these identified proteins.

Western blot analysis. The proteins levels of NADH dehydrogenase $1 \alpha$ subcomplex subunit 5 (Ndufa5), NADH dehydrogenase iron-sulfur protein 6 (Ndufs6), ATP synthase $\alpha$ subunit (ATP5A), transcription factor A, mitochondrial (TFAM) and cytochrome $b(\mathrm{CytB})$ from the liver mitochondria of the control and HFD groups were measured using western blot analysis. The proteins were extracted as described above in the 'Isolation of liver mitochondria' section and quantified by BCA Protein assay kit (Thermo Fisher Scientific, Inc.). The proteins $(20 \mu \mathrm{g})$ were separated on an SDS-PAGE gel and transferred onto nitrocellulose membranes (Bio-Rad Laboratories, Inc.), and then blocked with $5 \%$ nonfat milk buffer for $1.5 \mathrm{~h}$. The membranes were then incubated overnight with anti-Ndufa5 (1:1,000; cat. no. 16640-1-AP; ProteinTech Group, Inc., Chicago, USA), anti-Ndufs6 (1:1,000; cat. no 14417-1-AP; ProteinTech Group, Inc.), anti-ATP5A (1:1,000; cat. no. 14676-1-AP; ProteinTech
Table I. Body weights and liver weights or rats.

\begin{tabular}{lcc}
\hline Parameter & Con $(\mathrm{n}=6)$ & HFD $(\mathrm{n}=10)$ \\
\hline Initial body weight $(\mathrm{g})$ & $318.83 \pm 19.31$ & $321.30 \pm 15.25$ \\
Final body weight $(\mathrm{g})$ & $516.50 \pm 37.07$ & $548.20 \pm 22.23^{\mathrm{a}}$ \\
Liver weight $(\mathrm{g})$ & $11.71 \pm 1.17$ & $16.56 \pm 3.26^{\mathrm{b}}$ \\
Liver weight/body weight $(\%)$ & $2.26 \pm 0.22$ & $3.02 \pm 0.57^{\mathrm{b}}$ \\
\hline
\end{tabular}

Values are expressed as the mean \pm standard deviation. ${ }^{\mathrm{a}} \mathrm{P}<0.05$ and ${ }^{\mathrm{b}} \mathrm{P}<0.01$, vs. Con group. Con, control; HFD, high fat and high cholesterol diet.

Table II. Serum biochemical parameters.

\begin{tabular}{lcc}
\hline Parameter $(\mathrm{mM})$ & Con $(\mathrm{n}=6)$ & HFD $(\mathrm{n}=10)$ \\
\hline Glucose & $10.92 \pm 0.90$ & $10.49 \pm 2.10$ \\
TG & $0.56 \pm 0.13$ & $0.64 \pm 0.10$ \\
TC & $1.66 \pm 0.41$ & $2.44 \pm 0.48^{\mathrm{a}}$ \\
LDL-C & $1.07 \pm 0.35$ & $1.86 \pm 0.43^{\mathrm{a}}$ \\
HDL-C & $0.34 \pm 0.11$ & $0.29 \pm 0.13$
\end{tabular}

Values are expressed as the mean \pm standard deviation. ${ }^{\mathrm{a}} \mathrm{P}<0.01$, vs. Con. TC, total cholesterol; TG, triglyceride; LDL-C, low-density lipoprotein; HDL-C, high-density lipoprotein; Con, control; HFD, high fat and high cholesterol diet.

Group, Inc.), anti-TFAM (1:1,000; cat. no. ab131607; Abcam) and CytB (1:1,000; cat. no. 55090-1-AP; ProteinTech Group, Inc.) at $4^{\circ} \mathrm{C}$. The membranes were washed once with TBST buffer and incubated with horseradish peroxidase-conjugated anti-rabbit secondary antibodies (1:3,000; cat. no. A0208; Beyotime Institute of Biotechnology) at room temperature for $1 \mathrm{~h}$. The intensity of the bands was detected using ECL Western Blotting Substrate (Thermo Fisher Scientific, Inc.).

Statistical analysis. All results are presented as the mean \pm standard deviation and data were analyzed using Student's t-test. Statistical analyses were performed with SPSS 20.0 (IMB SPSS, Armonk, NY, USA). P<0.05 was considered to indicate a statistically significant difference.

\section{Results}

Characterization of the NASH rat model. The 16 weeks of HFD feeding successfully induced hepatic steatohepatitis. The body weights of the rats, compared with those in the control group, were significantly increased in the HFD group $(\mathrm{P}<0.05$; Table I). Of note, HFD chow resulted in a marked increase in the liver weight and hepatic index (liver weight/body weight \%). Higher levels $(\mathrm{P}<0.01)$ of serum TC and LDL-C were also found in the HFD-fed rats, compared with those in the controls, although no significant differences were found in the TG, HDL-C or glucose levels (Table II). Following 16 weeks of HFD feeding, the livers sections showed that there were abundant macrovesicular fat droplets 

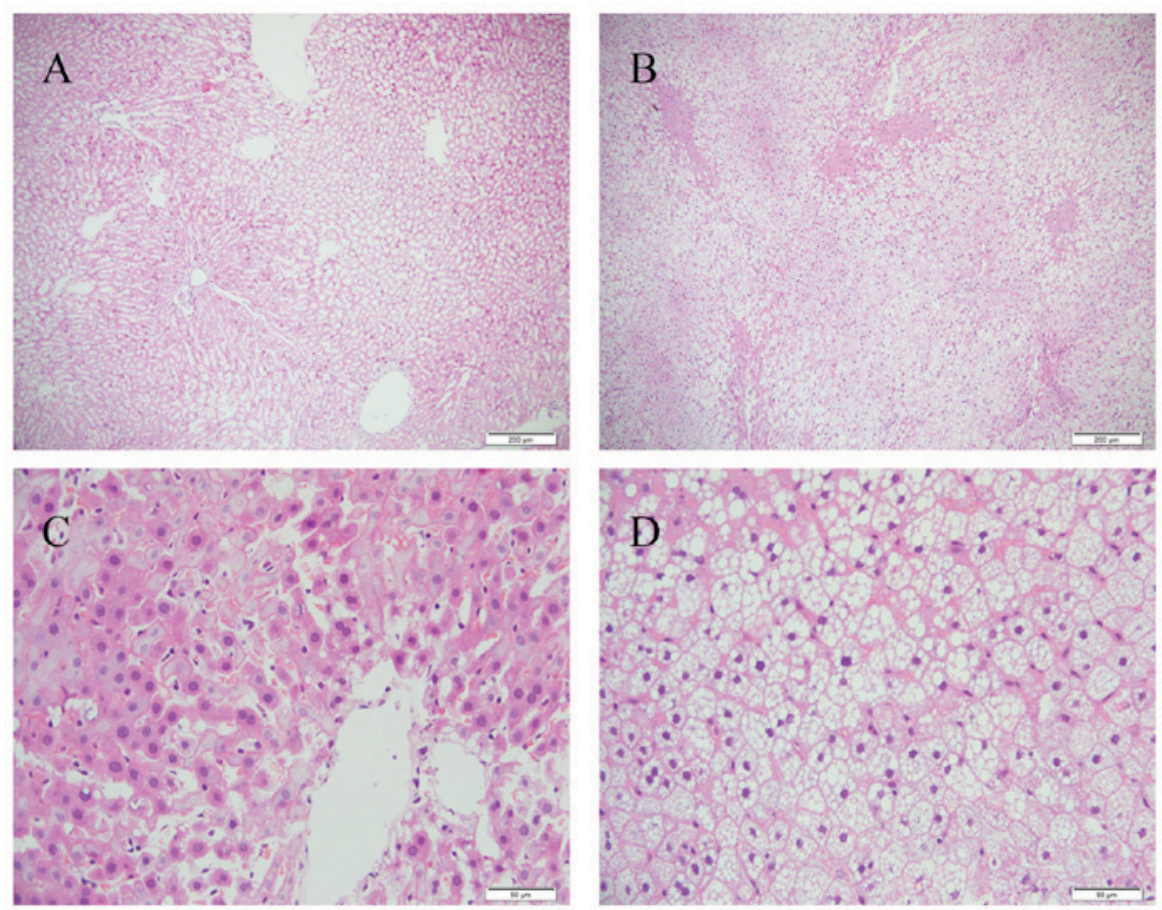

Figure 1. Hepatic pathology. Liver sections from the Con and HFD groups were stained with hematoxylin and eosin. Representative images from (A) Con and (B) HFD groups at x100 magnification. Representative images from (C) Con and (D) HFD groups at x400 magnification. Fat droplets, ballooned hepatocytes and inflammatory cells are present in the HFD group. HFD, high fat and high cholesterol diet; Con, control; HFD, high fat and high cholesterol diet.

in the hepatocytes and increased quantities of ballooned hepatocytes in the centrilobular parenchyma, leading to destruction of the normal structure of numerous hepatic lobules (Fig. 1). Furthermore, foci of necrosis and infiltration of inflammatory cells were identified in the centrilobular region. The liver tissues of the control group showed no histological abnormalities (Fig. 1).

Effect of mtDNA copy number. The present study also evaluated alterations of mitochondrial biogenesis in rats with HFD-induced NASH using information on mtDNA copy number. HFD feeding induced a significant decrease $(\mathrm{P}<0.05)$ in mtDNA copy number, which was reflected in the ratio of $\mathrm{ND}_{1}$ DNA to actin DNA in the liver (Fig. 2).

Evaluation of MRC and MMP. As MRCs are pivotal in the generation of energy by oxidative phosphorylation, the present study measured whether the activity of the MRC was affected in the liver tissues of the HFD group. As shown in Fig. 3A, the activity of complex I was significantly reduced in the HFD group, compared with that in the control group (complex I/CS, $1.93 \pm 0.56$ vs. $2.72 \pm 0.67 ; \mathrm{P}<0.05)$. No difference was observed in complex II (Fig. 3B), however, the activity of complex III was also significantly lower in the HFD group, compared with that in the control group (complex III/CS, $1.39 \pm 0.29$ vs. $1.81 \pm 0.24 ; \mathrm{P}<0.05)$, as shown in Fig. $3 \mathrm{C}$. No significant differences were observed in complex IV or ATP synthase (Fig. 3D and E). Citrate synthase is usually regarded as a quantitative marker enzyme for the content of intact mitochondria, and no significant change was detected between the HFD group and the control group (Fig. 3F). HFD chow also led to loss of MMP, which was associated with mitochondrial dysfunction (Fig. 4).

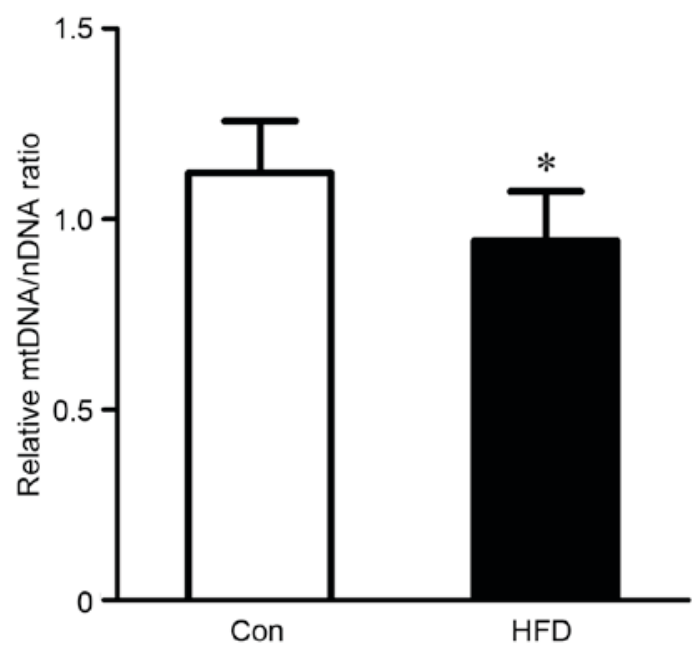

Figure 2. Evaluation of mtDNA copy numbers were evaluated in livers from rats in the Con and HFD groups via analysis of the ratio of $\mathrm{ND}_{1}$ DNA to actin DNA. The $\mathrm{ND}_{1}$ gene belongs to the mitochondrial genome, and actin gene belongs to the nuclear genome. Values are presented as the mean \pm standard deviation. ${ }^{*} \mathrm{P}<0.05$, vs. Con group. HFD, high fat and high cholesterol diet; Con, control; mtDNA, mitochondrial DNA.

Mitochondrial proteomic profiling analysis. In order to fully understand the pivotal role of mitochondria dysfunction in NASH, the present study measured the mitochondrial protein profiles of the liver from the HFD group model using iTRAQ labeling technology. A total of 61 significantly differentially expressed proteins were identified and were synchronous with the comparisons of liver mitochondrial samples isolated from the control and HFD groups. Compared with the control group, 30 upregulated proteins with a fold change $>1.0$ and 31 downregulated proteins 
A

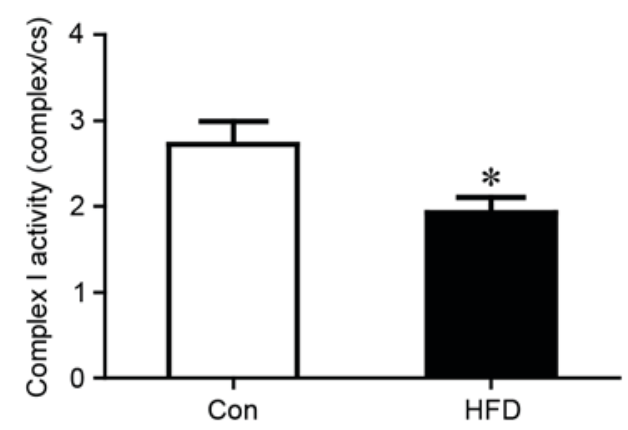

$\mathrm{C}$

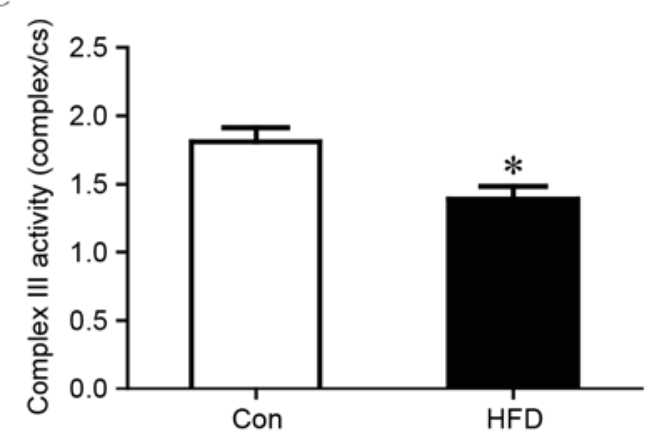

$\mathrm{E}$

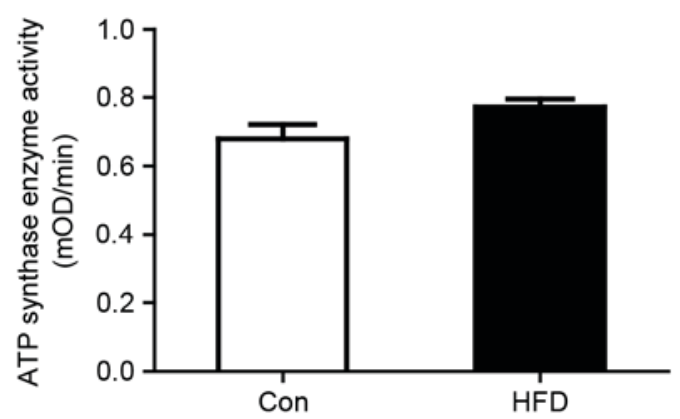

B

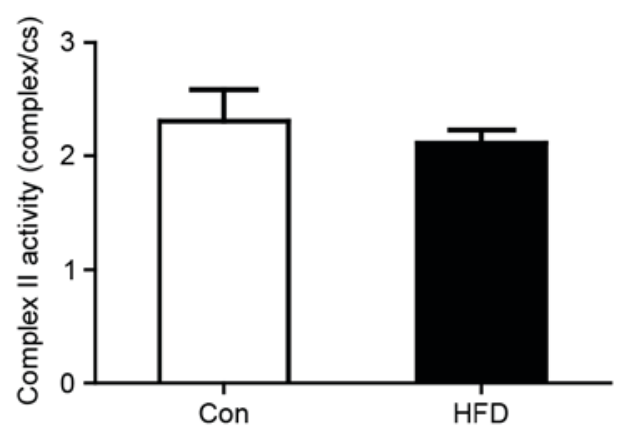

$\mathrm{D}$

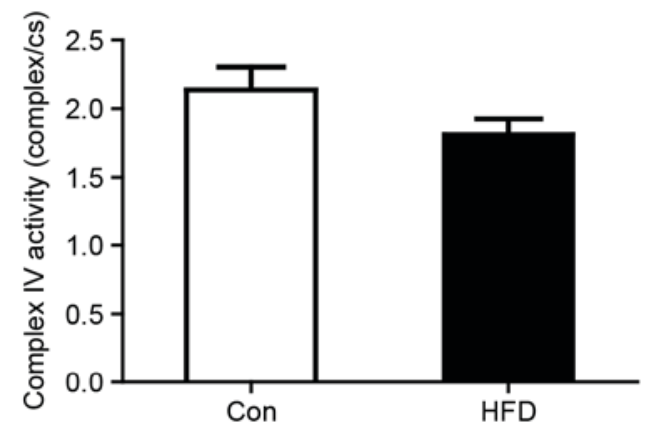

$\mathrm{F}$

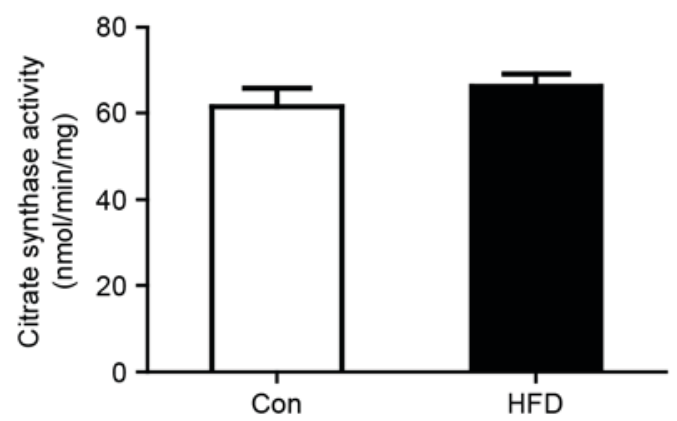

Figure 3. Analysis of mitochondrial respiratory chain enzymes in liver mitochondria isolated from control and HFD groups. Complexes (A) I, (B) II, (C) III, and (D) IV. Complex activities were normalized to CS. (E) ATP synthase activity (F) CS activity. The values are expressed as the mean \pm standard deviation. "P $<0.05$, vs. Con group. HFD, high fat and high cholesterol diet; Con, control; CS, citrate synthase.

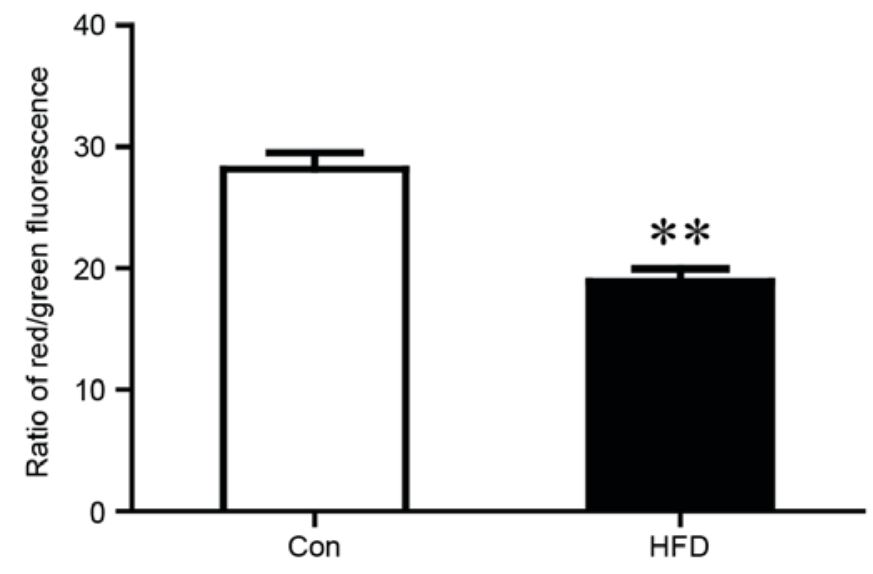

Figure 4. MMP was reflected by the ratio of red and green fluorescence. Liver mitochondria from the Con and HFD groups were isolated for MMP analysis. The values are presented as the mean \pm standard deviation. ${ }^{* *} \mathrm{P}<0.01$ vs. Con group. MMP, mitochondrial membrane potential; HFD, high fat and high cholesterol diet; Con, control. with a fold change $<1.0$ were observed in the HFD group, subsequently, 18 upregulated (Table III) with a fold change $>1.2$ and 13 downregulated proteins (Table IV) with a fold change $\leq 0.8$ were selected. The biological processes were found to be associated with dysregulated proteins using the GO database. Of note, the majority of these differentially expressed proteins were closely involved, including mitochondrial oxidative phosphorylation, lipid metabolic process, acyl-CoA metabolic process and fatty acid $\beta$-oxidation (Table V). In the HFD group, proteins involved in mitochondrial oxidative phosphorylation were almost unanimously downregulated, compared with the control group. Among the dysregulated proteins, seven decreased proteins, including Ndufc2, Ndufs6, Ndufb3, Ndufa2, Ndufa5, Ndufb5 and NADH-ubiquinone oxidoreductase chain $5\left(\mathrm{ND}_{5}\right)$, were component parts of mitochondrial complex II and the decreased proteins, ubiquinol-cytochrome c reductase, complex III subunit X (UQCR10) and cytochrome B (CytB) were the subunits of mitochondrial 
Table III. Identification of 18 mitochondrial proteins upregulated in the high fat and high cholesterol diet group, compared with the control group, using isobaric tags for relative and absolute quantitation labeling technology.

\begin{tabular}{|c|c|c|c|}
\hline Accession no. & Protein name & Gene name & Fold change \\
\hline trlQ5U2U5IQ5U2U5_RAT & Perilipin 2 & Plin2 & 3.61 \\
\hline splO70490|ACSM2_RAT & Acyl-coenzyme A synthetase & ACSM2 & 2.02 \\
\hline splP61354IRL27_RAT & 60S ribosomal protein L27 & Rpl27 & 1.94 \\
\hline splQ5EB77IRAB18_RAT & Ras-related protein Rab-18 & Rab18 & 1.90 \\
\hline trID3ZSY4ID3ZSY4_RAT & Eosinophil peroxidase & Epx & 1.68 \\
\hline trIF1LRE2|F1LRE2_RAT & $\begin{array}{l}\text { Insulin-like growth factor-binding protein complex } \\
\text { acid labile subunit }\end{array}$ & Igfals & 1.68 \\
\hline splP55159|PON1_RAT & Serum paraoxonase/arylesterase 1 & Pon1 & 1.64 \\
\hline splP11915INLTP_RAT & Non-specific lipid-transfer protein & Scp2 & 1.47 \\
\hline splP07687|HYEP_RAT & Epoxide hydrolase 1 & Ephx1 & 1.45 \\
\hline splP07871ITHIKB_RAT & 3-ketoacyl-CoA thiolase B & Acaa1b & 1.44 \\
\hline trlG3V743|G3V743_RAT & Glucosidase 1 & Mogs & 1.35 \\
\hline splQ5BJY9|K1C18_RAT & Keratin, type I cytoskeletal 18 & Krt18 & 1.32 \\
\hline trlD4ABM5ID4ABM5_RAT & Mitochondrial ribosomal protein S34 & Mrps34 & 1.31 \\
\hline splQ4FZX5IMSRB2_RAT & Methionine-R-sulfoxide reductase B2 & Msrb2 & 1.30 \\
\hline splP63095|GNAS2_RAT & Guanine nucleotide-binding protein $\mathrm{G}(\mathrm{s})$ subunit $\alpha$ isoforms & Gnas & 1.27 \\
\hline splP41034|TTPA_RAT & $\alpha$-tocopherol transfer protein & Ttpa & 1.25 \\
\hline splQ5U3Z3IISOC2_RAT & Isochorismatase domain-containing protein 2 & Isoc 2 & 1.24 \\
\hline trlF6Q5K7IF6Q5K7_RAT & Mitochondrial ribosomal protein S18B & Mrps18b & 1.22 \\
\hline
\end{tabular}

Fold-changes of $>1.2$ and $\mathrm{P}<0.05$ for all statistical data.

Table IV. Identification of 13 mitochondrial proteins downregulated in the high fat and high cholesterol diet group, compared with the control group, using isobaric tags for relative and absolute quantitation labeling technology.

\begin{tabular}{llcc}
\hline Accession no. & \multicolumn{1}{c}{ Protein name } & Gene name & Fold change \\
\hline trlF1LMQ2IF1LMQ2_RAT & Farnesyl pyrophosphate synthase & FPPS \\
splO35760IIDI1_RAT & Isopentenyl-diphosphate $\delta$-isomerase 1 & Idi1 \\
trlQ5PQZ9IQ5PQZ9_RAT & NADH dehydrogenase [ubiquinone] 1 subunit C2 & Ndufc2 \\
splP11951ICX6C2_RAT & Cytochrome $c$ oxidase subunit 6C-2 & Cox6c2 & 0.47 \\
trlB2RYX1IB2RYX1_RAT & Cytochrome b-c1 complex subunit 9 & 0.64 \\
trlD3ZCZ9ID3ZCZ9_RAT & NADH dehydrogenase [ubiquinone] iron-sulfur protein 6 & Uqcr10 & 0.65 \\
splQ63362INDUA5_RAT & NADH dehydrogenase [ubiquinone] 1 $\alpha$ subcomplex subunit 5 & Ndufa5 \\
trlD4A4P3ID4A4P3_RAT & NADH dehydrogenase [ubiquinone] 1 $\beta$ subcomplex subunit 3 & Ndufb3 & 0.65 \\
trlB0M1Q8IB0M1Q8_RAT & Cytochrome b & CytB \\
trID3ZS58ID3ZS58_RAT & NADH dehydrogenase [ubiquinone] 1 $\alpha$ subcomplex subunit 2 & 0.69 \\
trlD4A565ID4A565_RAT & NADH dehydrogenase (ubiquinone) 1 $\beta$ subcomplex, 5 & 0.72 \\
trlQ06QA1IQ06QA1_RAT & NADH-ubiquinone oxidoreductase chain 5 & 0.72 \\
splQ6PCT8IDHSD_RAT & Succinate dehydrogenase [ubiquinone] cytochrome b small subunit & Sdhd & 0.72 \\
\hline
\end{tabular}

Fold changes of $\leq 0.8$ and $\mathrm{P}<0.05$ for all statistical data.

complex III. In the inner mitochondrial membrane, these proteins form the middle segment of the respiratory chain. The decrease in these proteins coincided with the significant decline in the activities of complexes I and III (Fig. 3A and B). These proteomics may provide novel insights into pathogenesis of NASH, although further functional investigations are required to specify proteins.
Western blot analysis. The western blot analysis confirmed the differentially expressed proteins identified using iTRAQ technology. For the analysis, four proteins of interested were selected (Ndufa5, CytB, ATP5A and TFAM). Voltage-dependent anion-selective channel $1\left(\mathrm{VDAC}_{1}\right)$ was used as the loading control. The expression levels of Ndufa5 and $\mathrm{CytB}$ were significantly decreased in the HFD group, 
Table V. Dysregulated proteins involved in biological processes, according to the Gene Ontology database.

Biological process

Change in expression

Mitochondrial oxidative phosphorylation

$\mathrm{NADH}$ dehydrogenase [ubiquinone] 1 subunit $\mathrm{C} 2$

NADH dehydrogenase [ubiquinone] iron-sulfur protein 6

NADH dehydrogenase [ubiquinone] $1 \beta$ subcomplex subunit 3

NADH dehydrogenase [ubiquinone] $1 \alpha$ subcomplex subunit 2

NADH-ubiquinone oxidoreductase chain 5

NADH dehydrogenase [ubiquinone] $1 \alpha$ subcomplex subunit 5

Succinate dehydrogenase [ubiquinone] cytochrome $b$ small subunit

Cytochrome $b$

Cytochrome b-c1 complex subunit 9

Downregulated

Downregulated

Downregulated

Downregulated

Downregulated

Downregulated

Downregulated

Downregulated

Downregulated

Lipid metabolic process

Perilipin2

Upregulated

Acyl-coenzyme A synthetase

Upregulated

Arylsulfatase B

Upregulated

Estradiol 17- $\beta$-dehydrogenase 11

Upregulated

Non-specific lipid-transfer protein

Upregulated

3-ketoacyl-CoA thiolase B

Upregulated

Farnesyl pyrophosphate synthase

Downregulated

Isopentenyl-diphosphate $\delta$-isomerase 1

Downregulated

Cytochrome P450 2D18

Downregulated

Acyl-CoA-binding protein

Downregulated

Acyl-CoA metabolic process

Acyl-coenzyme A synthetase

Upregulated

Non-specific lipid-transfer protein

Upregulated

Acyl-CoA-binding protein

Downregulated

$\alpha$-aminoadipic semialdehyde synthase

Downregulated

Fatty acid $\beta$-oxidation

Non-specific lipid-transfer protein

Upregulated

3-ketoacyl-CoA thiolase B

Upregulated

compared with the control group, whereas no significant differences were observed in the levels of TFAM and ATP5A between the HFD group and control group. These findings were in accordance with the results obtained using iTRAQ technology (Fig. 5).

\section{Discussion}

NASH is considered to be important in the progression of NAFLD, which can progress into cirrhosis and subacute liver failure (1). However, the primary underlying mechanism contributing to the pathogenesis of NASH remains to be fully elucidated. The principal function of the mitochondria is to provide energy to maintain several cell functions. Previous studies have linked mitochondrial dysfunction and oxidative stress to the pathogenesis of NASH (7). Furthermore, mitochondrial structural abnormalities and decreased activity of MRCs have been reported in the livers of patients with NASH (13). In the present study, comprehensive analysis of liver mitochondrial proteomics was performed to provide a novel perspective for the pathophysiology of the condition.
In the proteomic analysis, a number of proteins involved in mitochondrial oxidative phosphorylation and the metabolism of lipids were significantly dysregulated, which may have contributed to the progression of NASH. For example, proteins in MRC complexes decreased significantly, including Ndufc2, Ndufs6, Ndufb3, Ndufa2, Ndufa5, Ndufb5, ND 5 (complex I), Sdhd (complex II), Cox6c2, Uqcr10 and CytB (complex III). Ndufc2 is one of the accessory subunits of complex I. The downregulation of Ndufc2 can decrease MMP and interfere with complex I integrity (13). The expression of Ndufb3 and Ndufb5, which are members of mitochondrial complex I, can be decreased by HFD, resulting in the dysfunction of mitochondrial oxidative phosphorylation in skeletal muscle (14). Ndufa5 localizes to the inner mitochondrial membrane, and its expression is reduced in the brains of patients with autism and in ischemic heart failure $(15,16)$, involving organs with high energy demand. The expression levels of $\mathrm{ND}_{5^{-}}$and cytochrome b-encoded mtDNA were decreased, which can be correlated with mtDNA depletion. The changes of 10 proteins in the mitochondrial complex was consistent with decreased activities of complex II and III. However, the downregulation of 
A

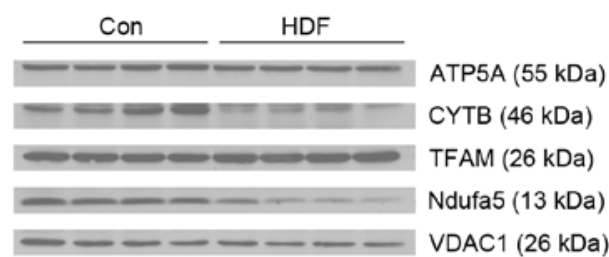

B

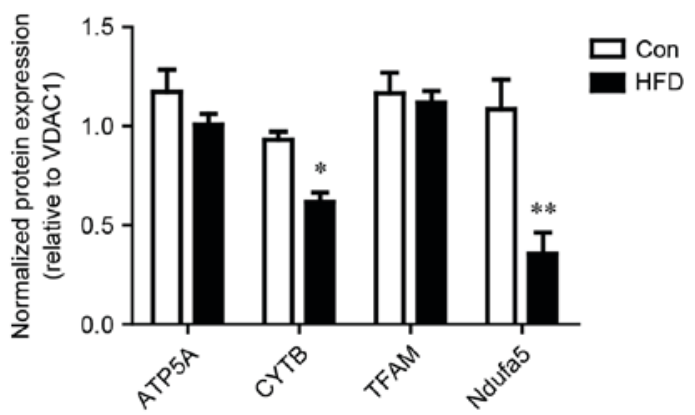

Figure 5. Western blot analysis validation of mitochondrial proteins. Liver mitochondrial expression levels of ATP5A, CytB, TFAM and Ndufa5 between the Con and HFD groups. VDAC $_{1}$ was used as a loading control. (A) Images of the western blots from four biological replicates. (B) Bar graphs of the results of the western blot analysis. The values are presented as the mean \pm standard deviation. ${ }^{*} \mathrm{P}<0.05$ and ${ }^{* *} \mathrm{P}<0.01$, vs. Con group. HFD, high fat and high cholesterol diet; Con, control; ATP5A, ATP synthase $\alpha$ subunit; CYTB, cytochrome b; TFAM, transcription factor A, mitochondrial; Ndufa5, NADH dehydrogenase [ubiquinone] $1 \alpha$ subcomplex subunit 5 ; $\mathrm{VDAC}_{1}$, voltage-dependent anion-selective channel 1.

Sdhd did not decrease the activity of complex II. Mitochondria complex I is critical in transferring electrons to ubiquinone. Complex I deficiency has been reported in several diseases, including heart failure, myopathies, encephalomyopathies and neurodegenerative disorders $(17,18)$. Furthermore, complex I and III are two sites of ROS generation in cells (19). It is well established that the administration of a HFD in rats leads to the profound modification of mitochondrial lipid composition, causes the inhibition of fatty acid oxidation and the generation of mitochondrial ROS (20). In terms of MRC activity, ROS are overproduced following any significant reduction, which triggers oxidative stress (7). ROS has been implicated in the hepatic tissue injury associated with NASH (5). The depletion of mtDNA in the HFD group may be associated with ROS, which leads to the degradation of mtDNA nucleases. Complex I abnormality may result in increasing ROS production, and ROS conversely affects the activity of mitochondrial complex I through the oxidative damage of cardiolipin (21), which can exacerbate oxidative stress and potentially contribute to the pathogenesis of NASH. In the present study, the decrease in components of complex I and III were expected to decrease complex activity with increasing ROS, which may be relevant to the decrease of MMP and be involved in the progression of NASH.

The present study also found several dysregulated proteins associated with preventing the progression of NASH. Non-specific lipid transfer protein (Scp2) is located in the cytoplasm and mitochondria (22). It has high affinity to several lipid species, including fatty acid, fatty acyl CoAs, lysophosphatidic acid, and phosphatidylinositol, and is also involved in the mitochondrial oxidation of cholesterol in cells (23).
Tumor necrosis factor and Fas, released by mitochondrial-free cholesterol, also induces NASH (24). Therefore, the upregulation of Scp2 may be a protective response to excess lipids in NASH. Medium-chain length fatty acids and xenobiotic carboxylic acids were catalyzed by the upregulation of acyl CoA synthetase (ACSM2) in the HFD group. In the liver, the decreased expression of ACSM2 can lead to an increase of free medium-chain fatty acids. Following treatment with medium-chain fatty acids, the protein level of the transcription factor, SREBP-1, is reduced and has a contrasting reaction to that of insulin in primary chicken hepatocytes (25). NASH is associated with insulin resistance, including lipolysis increase, free fatty acid delivery and hepatic fatty acid $\beta$ oxidation, which lead to an increase in oxidative stress (6). Therefore, ACSM2 may indirectly enhance insulin function in hepatocytes, which may be a protective response to insulin resistance in the liver. However, the specific generating process requires further investigation.

Among the downregulated proteins, farnesyl pyrophosphate synthase and Isopentenyl-diphosphate $\delta$-isomerase 1 are two enzymes of the mevalonate pathway, which catalyzes the synthesis of farnesyl pyrophosphate (FPP) in mitochondria (26). As with ubiquinones in the electron transport chain, mitochondrial isoprenoids are synthesized by FPP. However, the detailed association between FPP and the development of NASH remains to be fully elucidated.

In conclusion, the present study reports the protein profiling in NASH liver mitochondria using iTRAQ technology to perform detailed analysis of the pathogenesis of NASH for the first time. The results revealed 31 differentially expressed proteins in the NASH rat model, compared with the control rats. The dysregulation of proteins in NASH were predominantly associated with the MRC and lipid metabolic process. However, these proteins require further investigation for elucidating the molecular mechanism underlying the pathogenesis of NASH.

\section{Acknowledgements}

The present study was supported by a grant from the National Basic Research Program of China (grant no. 2013CB531702).

\section{References}

1. Patrick Melin AJ, Kalinski MI, Kelly KR, Haus JM, Solomon TP and Kirwan JP: Nonalcoholic fatty liver disease: Biochemical and therapeutic considerations. Ukr Biokhim Zh (1999) 81: 16-25, 2009.

2. Masuoka HC and Chalasani N: Nonalcoholic fatty liver disease: An emerging threat to obese and diabetic individuals. Ann N Y Acad Sci 1281: 106-122, 2013.

3. Day CP and James OF: Steatohepatitis: A tale of two 'hits'? Gastroenterology 114: 842-845, 1998.

4. Day CP: From fat to inflammation. Gastroenterology 130: 207-210, 2006.

5. Pessayre D and Fromenty B: NASH: A mitochondrial disease. J Hepatol 42: 928-940, 2005.

6. Sanyal AJ, Campbell Sargent C, Mirshahi F, Rizzo WB, Contos MJ, Sterling RK, Luketic VA, Shiffman ML and Clore JN: Nonalcoholic steatohepatitis: Association of insulin resistance and mitochondrial abnormalities. Gastroenterology 120: 1183-1192, 2001

7. Begriche K, Igoudjil A, Pessayre D and Fromenty B: Mitochondrial dysfunction in NASH: Causes, consequences and possible means to prevent it. Mitochondrion 6: 1-28, 2006. 
8. Anstee QM and Goldin RD: Mouse models in non-alcoholic fatty liver disease and steatohepatitis research. Int J Exp Pathol 87: $1-16,2006$

9. Ye H, Sun L, Huang X, Zhang $\mathrm{P}$ and Zhao X: A proteomic approach for plasma biomarker discovery with 8-plex iTRAQ labeling and SCX-LC-MS/MS. Mol Cell Biochem 343: 91-99, 2010.

10. Pallotti F and Lenaz G: Isolation and subfractionation of mitochondria from animal cells and tissue culture lines. Methods Cell Biol 80: 3-44, 2007.

11. Livak KJ and Schmittgen TD: Analysis of relative gene expression data using real-time quantitative PCR and the 2(-Delta Delta C(T)) method. Methods 25: 402-408, 2001.

12. Wieckowski MR, Giorgi C, Lebiedzinska M, Duszynski J and Pinton P: Isolation of mitochondria-associated membranes and mitochondria from animal tissues and cells. Nat Protoc 4 1582-1590, 2009.

13. Pérez Carreras M, Del Hoyo P, Martin MA, Rubio JC, Martin A, Castellano G, Colina F, Arenas J and Solis Herruzo JA: Defective hepatic mitochondrial respiratory chain in patients with nonalcoholic steatohepatitis. Hepatology 38: 999-1007, 2003.

14. Sparks LM, Xie H, Koza RA, Mynatt R, Hulver MW, Bray GA and Smith SR: A high-fat diet coordinately downregulates genes required for mitochondrial oxidative phosphorylation in skeletal muscle. Diabetes 54: 1926-1933, 2005.

15. Anitha A, Nakamura K, Thanseem I, Matsuzaki H, Miyachi T, Tsujii M, Iwata Y, Suzuki K, Sugiyama T and Mori N: Downregulation of the expression of mitochondrial electron transport complex genes in autism brains. Brain Pathol 23: 294-302, 2013

16. Liu T, Chen L, Kim E, Tran D, Phinney BS and Knowlton AA Mitochondrial proteome remodeling in ischemic heart failure. Life Sci 101: 27-36, 2014.

17. Kang PT, Chen CL, Ren P, Guarini G and Chen YR: BCNUinduced gR2 defect mediates S-glutathionylation of Complex I and respiratory uncoupling in myocardium. Biochem Pharmacol 89: 490-502, 2014
18. Finsterer J, Rauschka H, Segal L, Kovacs GG and Rolinski B: Affection of the respiratory muscles in combined complex I and IV deficiency. Open Neurol J 11: 1-6, 2017.

19. Murphy MP: How mitochondria produce reactive oxygen species. Biochem J 417: 1-13, 2009.

20. Vial G, Dubouchaud H, Couturier K, Cottet Rousselle C, Taleux N, Athias A, Galinier A, Casteilla L and Leverve XM: Effects of a high-fat diet on energy metabolism and ROS production in rat liver. J Hepatol 54: 348-356, 2011.

21. Paradies G, Petrosillo G, Pistolese M and Ruggiero FM: Reactive oxygen species affect mitochondrial electron transport complex I activity through oxidative cardiolipin damage. Gene 286: 135-141, 2002.

22. Gallegos AM, Atshaves BP, Storey SM, Starodub O, Petrescu AD, Huang H, McIntosh AL, Martin GG, Chao H, Kier AB and Schroeder F: Gene structure, intracellular localization, and functional roles of sterol carrier protein-2. Prog Lipid Res 40: 498-563, 2001

23. Schroeder F, Atshaves BP, McIntosh AL, Gallegos AM, Storey SM, Parr RD, Jefferson JR, Ball JM and Kier AB: Sterol carrier protein-2: New roles in regulating lipid rafts and signaling. Biochim Biophys Acta 1771: 700-718, 2007.

24. Mari M, Caballero F, Colell A, Morales A, Caballeria J, Fernandez A, Enrich C, Fernandez Checa JC and Garcia Ruiz C: Mitochondrial free cholesterol loading sensitizes to TNF- and Fas-mediated steatohepatitis. Cell Metab 4: 185-198, 2006.

25. Zhang Y, Yin L and Hillgartner FB: SREBP-1 integrates the actions of thyroid hormone, insulin, cAMP, and medium-chain fatty acids on ACCalpha transcription in hepatocytes. J Lipid Res 44: 356-368, 2003.

26. Martin D, Piulachs MD, Cunillera N, Ferrer A and Bellés X: Mitochondrial targeting of farnesyl diphosphate synthase is a widespread phenomenon in eukaryotes. Biochim Biophys Acta 1773: 419-426, 2007. 